\title{
Hyperfine induced electric dipole contributions to the electric octupole and magnetic quadrupole atomic clock transitions
}

\author{
V. A. Dzuba and V. V. Flambaum \\ School of Physics, University of New South Wales, Sydney 2052, Australia
}

(Dated: March 21, 2022)

\begin{abstract}
Hyperfine-induced electric dipole contributions may significantly increase probabilities of otherwise very weak electric octupole and magnetic quadrupole atomic clock transitions (e.g. transitions between $s$ and $f$ electron orbitals). These transitions can be used for exceptionally accurate atomic clocks, quantum information processing and search for dark matter. They are very sensitive to new physics beyond the Standard Model, such as temporal variation of the fine structure constant, the Lorentz invariance and Einstein equivalence principle violation. We formulate conditions under which the hyperfine-induced electric dipole contribution dominates. Due to the hyperfine quenching the electric octupole clock transition in ${ }^{173} \mathrm{Yb}^{+}$is two orders of magnitude stronger than that in currently used ${ }^{171} \mathrm{Yb}^{+}$. Some enhancement is found in ${ }^{143} \mathrm{Nd}^{13+},{ }^{149} \mathrm{Pm}^{14+},{ }^{147} \mathrm{Sm}^{14+}$, and ${ }^{147} \mathrm{Sm}^{15+}$ ions.

PACS numbers: 06.30.Ft, 06.20.Jr, 31.15.A, 32.30.Jc
\end{abstract}

Electric octupole (E3) and magnetic quadrupole (M2) atomic optical transitions, which correspond to transitions between $s$ and $f$ electron orbitals, can be used as optical clocks of exceptionally high accuracy [1-5]. They also present unique opportunities for fundamental research by being sensitive to new physics beyond the Standard Model. The transitions are very sensitive to the temporal variation of the fine structure constant $\alpha\left(\alpha=e^{2} / \hbar c\right)$ 2 10], to the local Lorentz invariance (LLI) violation [11], the effect of dark matter [12 18], etc. For example, the $4 f^{14} 6 s^{2} \mathrm{~S}_{1 / 2}-4 f^{13} 6 s^{2}{ }^{2} \mathrm{~F}_{7 / 2}^{o}$ transition in $\mathrm{Yb}^{+}$offers opportunities for frequency measurements with fractional accuracy $\sim 10^{-18}[1]$. The work is in progress in many laboratories 19 21]. Measuring the ratio of frequency of this transition to the frequency of the $4 f^{14} 6 s^{2} \mathrm{~S}_{1 / 2}-4 f^{14} 5 d^{2} \mathrm{D}_{3 / 2}$ transition in the same ion put the strongest limit on the temporal variation of the fine structure constant and (by including the Cs hyperfine transition) on the proton-to-electron mass ratio $[8,9,22$ 24]. The use of the electric octupole transition in $\mathrm{Yb}^{+}$ for the search of LLI violation may lead to five orders of magnitude improvement over current best bounds on the LLI violation in the electron-photon sector [11].

Many similar opportunities come with the use of optical transitions in highly-charged ions (HCI) [2 -5, 10]. For example, the spectrum of the $\operatorname{Ir}^{17+}$ ion has been recently measured 25] with the prospect of using the $4 f^{13} 5 s^{3} \mathrm{~F}_{4}^{o}-4 f^{12} 5 s^{2}{ }^{3} \mathrm{H}_{6}$ transition for the time keeping and fundamental research.

Electrical octupole and magnetic quadrupole transitions are very weak, typical linewidth can be as small as few $\mathrm{nHz}$. This may lead to certain difficulties in the measurements. In this paper we demonstrate that the electric dipole transition (E1) induced by hyperfine interaction can be significantly larger than the electric octupole or magnetic quadrupole transitions. Therefore, choosing right isotope might be important for the measurements.

At least one or more of the following conditions is needed for the domination of the hyperfine-induced E1 transitions.

- The hyperfine mixing is enhanced by a small energy interval. This is may be a fine structure interval for the ground or clock state. The clock transition in ${ }^{165} \mathrm{Ho}^{14+}$ ion [10] is an example.

- If $\Delta J<3$, the magnetic dipole hyperfine interaction (HFI) can contribute to the transition, where $J$ is the total electron angular momentum. Otherwise, an electric quadrupole HFI is needed, which is usually significantly smaller. For single-valenceelectron atoms or ions this would be a $4 f_{5 / 2}-5 s$ (or $6 s$ ) transition, e.g., the $4 f_{5 / 2}-5 s$ transition in ${ }^{143} \mathrm{Nd}^{13+}$. For two and more valence electrons, appropriated values of the total electron angular momentum are formed via contribution from all valence electrons.

- An isotope with a deformed nucleus has a large electric quadrupole moment. This makes the electric quadrupole HFI almost as large as the magnetic dipole HFI. The $4 f^{14} 6 s,{ }^{2} \mathrm{~S}_{1 / 2}-4 f^{13} 6 s^{2},{ }^{2} \mathrm{~F}_{7 / 2}^{o}$ transition in ${ }^{173} \mathrm{Yb}$ is an example.

- For smaller frequencies all transition rates are smaller. However, the electric octupole transition rate depends much stronger on $\omega\left(\sim \omega^{7}\right)$ than the electric dipole transition rate $\left(\sim \omega^{3}\right)$. Therefore, for a sufficiently small $\omega$ the electric dipole transition rate always dominates. The $4 f_{5 / 2}-5 s$ transition in ${ }^{149} \mathrm{Pm}^{14+}$ is an example.

The amplitude of the HFI-induced electric dipole transition is given by

$$
\begin{aligned}
& A_{\mathrm{hfs}-\mathrm{E} 1}(b \rightarrow a)= \\
& \sum_{n}\left[\frac{\left\langle a\left|\hat{\mathrm{H}}_{\mathrm{hfs}}\right| n\right\rangle\langle n|\hat{\mathrm{D}}| b\rangle}{E_{a}-E_{n}}+\frac{\left\langle b\left|\hat{\mathrm{H}}_{\mathrm{hfs}}\right| n\right\rangle\langle n|\hat{\mathrm{D}}| a\rangle}{E_{b}-E_{n}}\right] .
\end{aligned}
$$


Here $\hat{\mathrm{H}}_{\mathrm{hfs}}$ is the Hamiltonian of the magnetic dipole or electric quadrupole HFI, $\hat{D}$ is the electric dipole operator. The detailed expressions with the angular reduction can be found in Ref. 26, 27] and in the Appendix. The corresponding rate for the E1 transition (we use atomic units) is:

$$
R_{\mathrm{hfs}-\mathrm{E} 1}=\frac{4}{3}(\omega \alpha)^{3} \frac{A_{\mathrm{hfs}-\mathrm{E} 1}^{2}}{2 F_{c}+1},
$$

where $\omega$ is the frequency of the clock transition, $A_{\mathrm{hfs}-\mathrm{E} 1}$ is the amplitude of the transition (11) (the reduced matrix element), and $F_{c}$ is the total angular momentum of the clock state including the nuclear spin $I(\mathbf{F}=\mathbf{I}+\mathbf{J})$. The rate for a $\mathrm{E} 3$ transition is

$$
R_{\mathrm{E} 3}=0.00169(\omega \alpha)^{7} \frac{A_{\mathrm{E} 3}^{2}}{2 J_{c}+1},
$$

where $J_{c}$ is the total electron angular momentum of the clock state. The rate for a M2 transition is

$$
R_{\mathrm{M} 2}=\frac{1}{15}(\omega \alpha)^{5} \frac{A_{\mathrm{M} 2}^{2}}{2 J_{c}+1},
$$

where the amplitude of the magnetic quadrupole transition $A_{\mathrm{M} 2}$ includes the electron magnetic moment $\mu_{0}$ which is equal in the Gaussian atomic units to $\alpha / 2$.

We use the random phase approximation (RPA) to calculate the transition amplitudes and HFI mixing. The RPA equations for the core states $c$ are

$$
\left(\hat{H}^{\mathrm{HF}}-\epsilon_{c}\right) \delta \psi_{c}=-\left(\hat{F}+\delta V_{F}\right) \psi_{c},
$$

where $\hat{H}^{\mathrm{HF}}$ is the relativistic Hartree-Fock (HF) Hamiltonian, $\psi_{c}$ and $\epsilon_{c}$ are single-electron HF state in the core and its energy, $\left(\hat{H}^{\mathrm{HF}}-\epsilon_{c}\right) \psi_{c}=0, \delta \psi_{c}$ is the correction to the core state $c$ induced by an external field, $\hat{F}$ is the operator of the external filed, and $\delta V_{F}$ is the correction to the self-consistent HF potential induced by the external field via corrections to all core states. Equations (5) are solved self-consistently for all states in the core. Amplitude of the transition between valence states $v$ and $w$ (or mixing of these states) in the RPA approximation is given by the matrix element

$$
A_{w v}=\langle w|\hat{F}+\delta V| v\rangle .
$$

In present work we consider five different external field operators. These include the electric dipole and octupole operators, the magnetic quadrupole operator, the magnetic dipole and electric quadrupole HFI operators. Expressions for the single-electron matrix elements for each of these operators are presented in the Appendix.

In case of single-valence-electron atom or ion the amplitude (1) can be reduced to

$$
\begin{aligned}
& A_{\mathrm{hfs}-\mathrm{E} 1}(b \rightarrow a)= \\
& \left\langle\delta \psi_{a}\left|\hat{d}+\delta V_{d}\right| \psi_{b}\right\rangle+\left\langle\psi_{a}\left|\hat{d}+\delta V_{d}\right| \delta \psi_{b}\right\rangle,
\end{aligned}
$$

where $\delta \psi_{a}$ and $\delta \psi_{b}$ are corrections to valence states $a$ and $b$ induced by HFI, $\hat{d}=-e \mathbf{r}$ is the single-electron electric dipole operator, $\delta V_{d}$ is the correction to the HF potential of the core induced by the electric field of the photon.

In the case of several valence electrons we use the configuration interaction (CI) technique [9, 28] to construct many-electron states $a, b$ and $n$ in (11). Then we perform direct summation over excited states $n$. The summation is truncated at sufficiently high states so that the tail contribution is reasonably small.

A byproduct of this work is the calculated hyperfine structure of the ground and clock states of specific isotopes of several ions considered for the search of $\alpha$-variation [4, 5, 25, 34] and the Lorentz invariance violation [11]. This includes, e.g. the $\operatorname{Ir}^{17+}$ ion for which first measurements of the spectra were recently reported 25] confirming the predicted $5 s-4 f$ level crossing. The ${ }^{171} \mathrm{Yb}^{+}$ions are used by several experimental groups as prospective optical clocks of an exceptional high accuracy [20, 21, 23, 24, 35]. Current best limits on the temporal variation of the fine structure constant come from the comparison of the frequencies of the $4 f^{14} 6 s^{2} \mathrm{~S}_{1 / 2}-4 f^{13} 6 s^{2}{ }^{3} \mathrm{~F}_{2 / 2}$ E3 and $4 f^{12} 6 s^{2} \mathrm{~S}_{1 / 2}$ - $4 f^{14} 5 d^{2} \mathrm{D}_{3 / 2}$ E2 transitions [8, 9, 23, 24]. The results for the hyperfine structure are presented in Table【 Apart from getting new data, the comparison with the experiment for ${ }^{171} \mathrm{Yb}^{+}$and ${ }^{173} \mathrm{Yb}^{+}$tests the accuracy of the calculations. It indicates that the theoretical uncertainty is smaller than $10 \%$.

Table Ipresents calculated E3 and M2 transition rates for the clock states of the ions. They are to be compared with the HFI-induced rates in Table III] The calculated E3 transition rate for $\mathrm{Yb}^{+}$is within error bars of the experimental value [1]. The E3 transition rate is larger than the M2 rate in all ions except for the $\mathrm{Sm}^{14+}$ ion, where it is small due to the small frequency.

The calculated HFI-induced E1 transition rates are presented in Table III]. They are larger than the E3 or M2 rates for the ${ }^{143} \mathrm{Nd}^{13+},{ }^{149} \mathrm{Pm}^{14+},{ }^{147} \mathrm{Sm}^{14+}$, and ${ }^{173} \mathrm{Yb}^{+}$ions (see Table II). The most interesting case is probably the ${ }^{173} \mathrm{Yb}^{+}$ion. This is due to its importance as one of the most accurate optical clocks, and study of the temporal variation of fine structure constant and the Lorentz invariance violation as discussed above. Currently used ${ }^{171} \mathrm{Yb}^{+}$ion has a very weak clock transition rate, about two orders of magnitude weaker than the rate in the ${ }^{173} \mathrm{Yb}^{+}$ion calculated in the present work. This is due to the HFI-induced E1 transition in ${ }^{173} \mathrm{Yb}^{+}$which is relatively large due to the large contribution from the electric quadrupole hyperfine mixing. The ${ }^{171} \mathrm{Yb}^{13+}$ isotope has small nuclear spin $(I=1 / 2)$ and no nuclear electric quadrupole moment.

The main reason for using odd isotope for the E3 clock transition in $\mathrm{Yb}^{+}$is the possibility to eliminate linear Zeeman shift by using states with $M=0$ [1] ( $M$ is the projection of the total angular momentum of the atom, including nuclear spin). This works for both ${ }^{171} \mathrm{Yb}^{+}$and ${ }^{173} \mathrm{Yb}^{+}$. Note that the electric quadrupole shift due to 
TABLE I: Hyperfine structure of the ground and clock states of the ions considered in this work. $I$ is the nuclear spin, $\mu$ is the nuclear magnetic dipole moment in nuclear magnetons, $Q$ is the nuclear electric quadrupole moment in barns $\left(10^{-28} \mathrm{~m}^{2}\right)$, $A$ is the magnetic dipole hfs constant (in $\mathrm{MHz}$ ), $B$ is the electric quadrupole hfs constant (in $\mathrm{MHz}$ ). A comparison with an experiment is given for $\mathrm{Yb}^{+}$.

\begin{tabular}{|c|c|c|c|c|c|c|c|c|c|c|c|}
\hline \multirow{2}{*}{$\begin{array}{c}\text { Ion } \\
\text { Isotope } \\
{ }^{143} \mathrm{Nd}^{13+}\end{array}$} & $I$ & \multirow{2}{*}{$\begin{array}{c}\mu \\
\mu_{N} \\
-1.08\end{array}$} & \multirow{2}{*}{$\begin{array}{c}Q \\
\mathrm{~b} \\
-0.630(60)\end{array}$} & \multicolumn{2}{|c|}{$\begin{array}{c}\text { Ground } \\
\text { State }\end{array}$} & \multirow{2}{*}{$\begin{array}{c}A \\
\mathrm{MHz} \\
-38200\end{array}$} & \multirow{2}{*}{$\begin{array}{c}\begin{array}{c}B \\
\mathrm{MHz}\end{array} \\
0\end{array}$} & \multicolumn{2}{|c|}{$\begin{array}{l}\text { Clock } \\
\text { State }\end{array}$} & \multirow{2}{*}{$\begin{array}{c}A \\
\mathrm{MHz} \\
-333\end{array}$} & \multirow{2}{*}{$\begin{array}{c}\begin{array}{c}B \\
\mathrm{MHz}\end{array} \\
-833\end{array}$} \\
\hline & $7 / 2$ & & & $5 s$ & ${ }^{2} \mathrm{~S}_{1 / 2}$ & & & $4 f$ & ${ }^{2} \mathrm{~F}_{5 / 2}^{o}$ & & \\
\hline${ }^{149} \mathrm{Pm}^{14+}$ & $7 / 2$ & \pm 3.3 & no data & $5 s$ & ${ }^{2} \mathrm{~S}_{1 / 2}$ & \pm 130500 & 0 & $4 f$ & ${ }^{2} \mathrm{~F}_{5 / 2}^{o}$ & \pm 1162 & $1530^{a}$ \\
\hline${ }^{147} \mathrm{Sm}^{15+}$ & $7 / 2$ & -0.813 & $-0.259(26)$ & $4 f$ & ${ }^{2} \mathrm{~F}_{5 / 2}^{O}$ & -318 & -443 & $5 s$ & ${ }^{2} \mathrm{~S}_{1 / 2}$ & -34800 & 0 \\
\hline${ }^{147} \mathrm{Sm}^{14+}$ & $7 / 2$ & -0.813 & $-0.259(26)$ & $4 f^{2}$ & ${ }^{3} \mathrm{H}_{4}$ & -320 & 3728 & $5 s 4 f$ & ${ }^{3} \mathrm{~F}_{2}^{o}$ & 5222 & 2833 \\
\hline${ }^{193} \operatorname{Ir}^{17+}$ & $3 / 2$ & 0.1591 & $0.751(9)$ & $4 f^{13} 5 s$ & ${ }^{3} \mathrm{~F}_{4}^{o}$ & 5180 & -3218 & $4 f^{12} 5 s^{2}$ & ${ }^{3} \mathrm{H}_{6}$ & 176 & -3332 \\
\hline${ }^{171} \mathrm{Yb}^{+}$ & $1 / 2$ & 0.4919 & 0 & $4 f^{14} 6 s$ & ${ }^{2} \mathrm{~S}_{1 / 2}$ & 11600 & 0 & $4 f^{13} 6 s^{2}$ & ${ }^{3} \mathrm{~F}_{7 / 2}^{o}$ & 871 & 0 \\
\hline${ }^{171} \mathrm{Yb}^{+}$ & \multicolumn{2}{|c|}{ Experiment $^{b}$} & & & & 12645 & & & & $905.0(5)$ & \\
\hline${ }^{173} \mathrm{Yb}^{+}$ & $5 / 2$ & -0.6776 & $2.800(4)$ & $4 f^{14} 6 s$ & ${ }^{2} \mathrm{~S}_{1 / 2}$ & -3200 & 0 & $4 f^{13} 6 s^{2}$ & ${ }^{3} \mathrm{~F}_{7 / 2}^{o}$ & -240 & -4762 \\
\hline${ }^{173} \mathrm{Yb}^{+}$ & \multicolumn{2}{|c|}{ Experiment $^{c}$} & & & & $-3497.5(6)$ & & & & & \\
\hline
\end{tabular}

${ }^{a}$ Assuming $Q=1 \mathrm{~b}$.

${ }^{b}$ Ref. 29].

${ }^{c}$ Ref. [30].

TABLE II: Experimental or theoretical frequencies of the clock transitions $(\omega)$ and theoretical rates of the spontaneous decay of the clock state due to the electric octupole (E3), or magnetic quadrupole (M2) transitions to the ground state for the ions of Table I. The experimental rate for $\mathrm{Yb}^{+}$is $0.59(+1.21 /-0.38) \times 10^{-8} \mathrm{~s}^{-1}[1]$.

\begin{tabular}{|c|c|c|c|c|c|c|c|}
\hline Ion & $\begin{array}{l}\text { Gro } \\
\text { St }\end{array}$ & $\begin{array}{l}\text { und } \\
\text { ate }\end{array}$ & $\begin{array}{l}\text { Cloc } \\
\text { Stat }\end{array}$ & & $\begin{array}{c}\omega \\
\mathrm{cm}^{-1}\end{array}$ & $\begin{array}{l}R_{\mathrm{E} 3} \\
\mathrm{~s}^{-1}\end{array}$ & $\begin{array}{l}R_{\mathrm{M} 2} \\
\mathrm{~s}^{-1}\end{array}$ \\
\hline $\mathrm{Nd}^{13}$ & $5 s$ & ${ }^{2} \mathrm{~S}_{1 / 2}$ & $\overline{4 f}$ & ${ }^{2} \mathrm{~F}_{5}^{\mathrm{c}}$ & $55870^{a}$ & $8.0[-7]$ & $4.6[-11]$ \\
\hline $\mathrm{Pm}^{14+}$ & $5 s$ & ${ }^{2} \mathrm{~S}_{1 / 2}$ & $4 f$ & ${ }^{2} \mathrm{~F}_{5 / 2}^{o}$ & $3228^{b}$ & $1.2[-14]$ & $2.5[-17]$ \\
\hline $\mathrm{Sm}^{15+}$ & $4 f$ & ${ }^{2} \mathrm{~F}_{5 / 2}^{o}$ & $5 s$ & ${ }^{2} \mathrm{~S}_{1 / 2}$ & $60384^{a}$ & $2.2[-6]$ & $1.1[-8]$ \\
\hline $\mathrm{Sm}^{14+}$ & $4 f^{2}$ & ${ }^{3} \mathrm{H}_{4}$ & $5 s 4 f$ & ${ }^{3} \mathrm{~F}_{2}^{o}$ & $2172^{c}$ & $1.5[-16]$ & $1.5[-14]$ \\
\hline $\operatorname{Ir}^{17+}$ & $4 f^{13} 5 s$ & ${ }^{3} \mathrm{~F}_{4}^{o}$ & $4 f^{12} 5 s^{2}$ & ${ }^{3} \mathrm{H}_{6}$ & $37423^{d}$ & $7.9[-9]$ & $3.0[-9]$ \\
\hline $\mathrm{Yb}^{+}$ & $4 f^{14} 6 s$ & ${ }^{2} \mathrm{~S}_{1 / 2}$ & $4 f^{13} 6 s^{2}$ & ${ }^{3} \mathrm{~F}_{7 / 2}^{o}$ & $21419^{e}$ & $1.0[-8]$ & {$\left[\begin{array}{ll}1 & 0\end{array}\right.$} \\
\hline
\end{tabular}

${ }^{a}$ Expt., 31].

${ }^{b}$ Theory, this work.

${ }^{c}$ Theory, 32].

${ }^{d}$ Expt. 25.

${ }^{e}$ Expt. 33].

gradients of electric field can also be eliminated in both isotopes by considering states with $F=3, M= \pm 2$ $(\mathbf{F}=\mathbf{J}+\mathbf{I}, M$ is projection of $\mathbf{F}$, the energy shift $\Delta E \sim$ $3 M^{2}-F(F+1)$ vanishes for $\left.F=3, M= \pm 2\right)$. In this case the linear Zeeman shift can be eliminated by averaging the measurements involving states with $M=+2$ and $M=-2$.

\section{Acknowledgments}

The work was supported in part by the Australian Research Council.

\section{Appendix A: Matrix elements}

The matrix element of the electromagnetic transition amplitude between many-electron states with definite values of the electron total angular momentum $J$ and the total atomic angular momentum $F(\mathbf{F}=(J)+\mathbf{I}$, where $I$ is the nuclear spin) is given by

$$
\begin{aligned}
& \left\langle J_{a}, F_{a}\left\|A_{k}\right\| J_{b}, F_{b}\right\rangle=(-1)^{I+F_{b}+J_{a}+k} \times \\
& \sqrt{\left(2 F_{a}+1\right)\left(2 F_{b}+1\right)}\left\{\begin{array}{lll}
J_{b} & J_{a} & k \\
F_{a} & F_{b} & I
\end{array}\right\}\left\langle J_{a}\left\|A_{k}\right\| J_{b}\right\rangle,
\end{aligned}
$$

where $k$ is the operator $\operatorname{rank}(k=1$ for E1 and M1, $k=2$ for $\mathrm{E} 2$ and M2, etc.).

The matrix element of the magnetic dipole HFI is

$$
\begin{aligned}
& \left\langle J_{a}, F\left\|\hat{H}_{A}\right\| J_{b}, F\right\rangle=(-1)^{I+F+J_{b}} \times \\
& \mu \sqrt{\frac{(2 I+1)(I+1)}{I}}\left\{\begin{array}{ccc}
I & J_{b} & F \\
J_{a} & I & 1
\end{array}\right\}\left\langle J_{a}\left\|\hat{H}_{A}\right\| J_{b}\right\rangle .
\end{aligned}
$$

Here $\mu$ is the nuclear magnetic dipole moment in nuclear magnetons.

The matrix element of the electric quadrupole HFI is

$$
\begin{aligned}
& \left\langle J_{a}, F\left\|\hat{H}_{B}\right\| J_{b}, F\right\rangle=\frac{Q}{2}(-1)^{I+F+J_{b}} \times \\
& \sqrt{\frac{(I+1)(2 I+1)(2 I+3)}{I(2 I-1)}}\left\{\begin{array}{ccc}
I & J_{b} & F \\
J_{a} & I & 2
\end{array}\right\}\left\langle J_{a}\left\|\hat{H}_{B}\right\| J_{b}\right\rangle .
\end{aligned}
$$

Here $Q$ is the nuclear electric quadrupole moment in atomic units.

In expressions (A1), (A2) and (A3) the matrix element $\left\langle J_{a}|| \hat{H}|| J_{b}\right\rangle$ is the matrix element of a one-body operator between many-electron CI wave functions of valence electrons. The standard CI technique is used to reduce theses matrix elements to the single-electron matrix elements. 
TABLE III: The rate of the HFI-induced electric dipole transitions between hyperfine components of the ground and clock states of the ions for Table \

\begin{tabular}{|c|c|c|c|c|}
\hline Ion & Clock & & & $R_{\mathrm{E} 1-\mathrm{HFI}}$ \\
\hline Isotope & Transition & $F_{g}$ & $F_{c}$ & $\mathrm{~s}^{-1}$ \\
\hline \multirow[t]{6}{*}{${ }^{143} \mathrm{Nd}^{13+}$} & ${ }^{2} \mathrm{~S}_{1 / 2}-{ }^{2} \mathrm{~F}_{5 / 2}^{o}$ & 3.0 & 2.0 & $7.2[-8]$ \\
\hline & & 3.0 & 3.0 & $1.1[-7]$ \\
\hline & & 3.0 & 4.0 & $8.2[-8]$ \\
\hline & & 4.0 & 3.0 & $3.7[-8]$ \\
\hline & & 4.0 & 4.0 & $1.1[-7]$ \\
\hline & & 4.0 & 5.0 & $1.7[-7]$ \\
\hline \multirow{6}{*}{${ }^{149} \mathrm{Pm}^{14+}$} & ${ }^{2} \mathrm{~S}_{1 / 2}-{ }^{2} \mathrm{~F}_{5 / 2}^{o}$ & 3.0 & 2.0 & $4.8[-11]$ \\
\hline & & 3.0 & 3.0 & $7.4[-11]$ \\
\hline & & 3.0 & 4.0 & $5.5[-11]$ \\
\hline & & 4.0 & 3.0 & $2.5[-11]$ \\
\hline & & 4.0 & 4.0 & $7.6[-11]$ \\
\hline & & 4.0 & 5.0 & $1.1[-10]$ \\
\hline \multirow{6}{*}{${ }^{147} \mathrm{Sm}^{15+}$} & ${ }^{2} \mathrm{~F}_{5 / 2}^{o}-{ }^{2} \mathrm{~S}_{1 / 2}$ & 2.0 & 3.0 & $5.0[-9]$ \\
\hline & & 3.0 & 3.0 & $1.1[-8]$ \\
\hline & & 3.0 & 4.0 & $2.9[-9]$ \\
\hline & & 4.0 & 3.0 & $1.0[-8]$ \\
\hline & & 4.0 & 4.0 & $1.1[-8]$ \\
\hline & & 5.0 & 4.0 & $2.0[-8]$ \\
\hline \multirow[t]{15}{*}{${ }^{147} \mathrm{Sm}^{14+}$} & ${ }^{3} \mathrm{H}_{4}-{ }^{3} \mathrm{~F}_{2}^{o}$ & 0.5 & 1.5 & $6.1[-10]$ \\
\hline & & 1.5 & 1.5 & $2.8[-9]$ \\
\hline & & 1.5 & 2.5 & $1.2[-9]$ \\
\hline & & 2.5 & 1.5 & $2.9[-9]$ \\
\hline & & 2.5 & 2.5 & $5.9[-9]$ \\
\hline & & 2.5 & 3.5 & $1.4[-9]$ \\
\hline & & 3.5 & 2.5 & $7.8[-9]$ \\
\hline & & 3.5 & 3.5 & $7.9[-9]$ \\
\hline & & 3.5 & 4.5 & $1.0[-9]$ \\
\hline & & 4.5 & 3.5 & $1.4[-8]$ \\
\hline & & 4.5 & 4.5 & $7.5[-9]$ \\
\hline & & 4.5 & 5.5 & $4.1[-10]$ \\
\hline & & 5.5 & 4.5 & $1.9[-8]$ \\
\hline & & 5.5 & 5.5 & $4.3[-9]$ \\
\hline & & 6.5 & 5.5 & $1.7[-8]$ \\
\hline \multirow[t]{6}{*}{${ }^{193} \operatorname{Ir}^{17+}$} & ${ }^{3} \mathrm{~F}_{4}^{o}-{ }^{3} \mathrm{H}_{6}$ & 3.5 & 4.5 & $5.0[-9]$ \\
\hline & & 4.5 & 4.5 & $4.7[-10]$ \\
\hline & & 5.5 & 4.5 & $6.7[-12]$ \\
\hline & & 4.5 & 5.5 & $3.8[-10]$ \\
\hline & & 5.5 & 5.5 & $2.2[-11]$ \\
\hline & & 5.5 & 6.5 & $4.0[-9]$ \\
\hline \multirow[t]{6}{*}{${ }^{173} \mathrm{Yb}^{+}$} & ${ }^{2} \mathrm{~S}_{1 / 2}-{ }^{3} \mathrm{~F}_{7 / 2}^{o}$ & 2.0 & 1.0 & $3.3[-7]$ \\
\hline & & 2.0 & 2.0 & $1.8[-6]$ \\
\hline & & 3.0 & 2.0 & $5.1[-7]$ \\
\hline & & 2.0 & 3.0 & $2.5[-6]$ \\
\hline & & 3.0 & 3.0 & $3.2[-6]$ \\
\hline & & 3.0 & 4.0 & $6.5[-6]$ \\
\hline
\end{tabular}

We use single-electron wave functions in a form

$$
\psi(r)_{n j l m}=\frac{1}{r}\left(\begin{array}{c}
f_{v}(r) \Omega(\mathbf{n})_{j l m} \\
i \alpha g_{v}(r) \widetilde{\Omega}(\mathbf{n})_{j l m}
\end{array}\right),
$$

where $n$ is the principal quantum number and the in$\operatorname{dex} v$ replaces the three-number set $n, j, l ; \alpha$ is the fine structure constant.

The matrix elements of the electric multipole transitions are $(\alpha \omega r \ll 1)$

$$
\begin{aligned}
& \left\langle a|| E_{k}|| b\right\rangle=\left\langle\kappa_{a}\left\|C_{k}\right\| \kappa_{b}\right\rangle \times \\
& \int\left(f_{a}(r) f_{b}(r)+\alpha^{2} g_{a}(r) g_{b}(r)\right) r^{k} d r
\end{aligned}
$$

The matrix elements of the magnetic quadrupole transitions are

$$
\begin{aligned}
& \left\langle a|| M_{2}|| b\right\rangle=\left\langle-\kappa_{a}\left\|C_{2}\right\| \kappa_{b}\right\rangle\left(\kappa_{a}+\kappa_{b}\right) \times \\
& \frac{2 \alpha}{3} \int\left(f_{a}(r) g_{b}(r)+g_{a}(r) f_{b}(r)\right) r^{2} d r
\end{aligned}
$$

The matrix elements of the magnetic dipole HFI are

$$
\begin{aligned}
& \left\langle a|| \hat{H}_{A} \| b\right\rangle=-\left\langle-\kappa_{a}\left\|C_{1}\right\| \kappa_{b}\right\rangle\left(\kappa_{a}+\kappa_{b}\right) \times \\
& \frac{\alpha^{2}}{2} \frac{m_{e}}{m_{p}} \int\left(f_{a}(r) g_{b}(r)+g_{a}(r) f_{b}(r)\right) \frac{r}{r_{>}^{3}} d r
\end{aligned}
$$

The matrix elements of the electric quadrupole HFI are

$$
\begin{aligned}
& \left\langle a\left\|\hat{H}_{B}\right\| b\right\rangle=\left\langle\kappa_{a}\left\|C_{2}\right\| \kappa_{b}\right\rangle \times \\
& \int\left(f_{a}(r) f_{b}(r)+\alpha^{2} g_{a}(r) g_{b}(r)\right) \frac{r}{r_{>}^{4}} d r
\end{aligned}
$$

In (A7) and (A8) $r_{>}=\max \left(r, r_{N}\right)$, where $r_{N}$ is the nuclear radius.

The reduced matrix element of the spherical harmonic $C_{k}$ is

$$
\begin{gathered}
\left\langle\kappa_{a}\left\|C_{k}\right\| \kappa_{b}\right\rangle=(-1)^{j_{b}+1 / 2} \sqrt{\left(2 j_{a}+1\right)\left(2 j_{b}+1\right)} \\
\times \xi\left(l_{a}+l_{b}+k\right)\left(\begin{array}{ccc}
j_{b} & j_{a} & k \\
-1 / 2 & 1 / 2 & 0
\end{array}\right) . \\
\xi(x)=\left\{\begin{array}{llll}
1, & \text { if } x & \text { is even } \\
0, & \text { if } x & \text { is } & \text { odd }
\end{array}\right.
\end{gathered}
$$

[1] M. Roberts, P. Taylor, G. P. Barwood, W. R. C. Rowley, and P. Gill, Phys. Rev. A 62, 020501 (2000).

[2] J. C. Berengut, V. A. Dzuba, and V. V. Flambaum, Phys. Rev. Lett. 105, 120801 (2010).

[3] J. C. Berengut, V. A. Dzuba, V. V. Flambaum, and A. Ong, Phys. Rev. Lett. 106, 210802 (2011).

[4] V. A. Dzuba, A. Derevianko, and V. V. Flambaum, Phys. Rev. A 86, 054502 (2012).
[5] M. S. Safronova, V. A. Dzuba, V. V. Flambaum, U. I. Safronova, S. G. Porsev, and M. G. Kozlov, Phys. Rev. Lett. 113, 030801 (2014).

[6] V. A. Dzuba, V. V. Flambaum, and J. K. Webb, Phys. Rev. Lett. 82, 888 (1999).

[7] V. A. Dzuba, V. V. Flambaum, and J. K. Webb, Phys. Rev. A 59, 230 (1999).

[8] V. A. Dzuba, V. V. Flambaum, and M. V. Marchenko, 
Phys. Rev. A 68, 022506 (2003).

[9] V. A. Dzuba and V. V. Flambaum, Phys. Rev. A 77, 012515 (2008).

[10] V. A. Dzuba, V. V. Flambaum, and H. Katori, Phys. Rev. A 91, 022119 (2015).

[11] V. A. Dzuba, V. V. Flambaum, M. S. Safronova, S. G. Porsev, T. Pruttivarasin, M. A. Hohensee, and H. Häffner, Nature Physics (2016).

[12] P. W. Graham and S. Rajendran, Phys. Rev. D 88, 035023 (2013).

[13] D. Budker, P. W. Graham, M. Ledbetter, S. Rajendran, and A. O. Sushkov, Phys. Rev. X 4, 021030 (2014).

[14] K. Van Tilburg, N. Leefer, L. Bougas, and D. Budker, Phys. Rev. Lett. 115, 011802 (2015).

[15] Y. V. Stadnik and V. V. Flambaum, Phys. Rev. D 89, 043522 (2014).

[16] Y. V. Stadnik and V. V. Flambaum, Phys. Rev. Lett. 113, 151301 (2014).

[17] Y. V. Stadnik and V. V. Flambaum, Phys. Rev. Lett. 114, 161301 (2015).

[18] Y. V. Stadnik and V. V. Flambaum, Phys. Rev. Lett. 115, 201301 (2015).

[19] C. Tamm, S. Weyers, B. Lipphardt, and E. Peik, Phys. Rev. A 80, 043403 (2009).

[20] S. A. King, R. M. Godun, S. A. Webster, H. S. Margolis, L. A. M. Johnson, K. Szymaniec, P. E. G. Baird, and P. Gill, New J. Phys. 14, 013045 (2012).

[21] A. Rastogi, N. Batra, A. Roy, J. Thangjam, V. P. S. Kalsi, S. Panja, and S. De, MAPAN 30, 169 (2015).

[22] V. V. Flambaum and A. F. Tedesco, Phys. Rev. C 73, 055501 (2006).

[23] R. M. Godun, P. B. R. Nisbet-Jones, J. M. Jones, S. A. King, L. A. M. Johnson, H. S. Margolis, K. Szymaniec, S. N. Lea, K. Bongs, and P. Gill, Phys. Rev. Lett. 113, 210801 (2014).
[24] N. Huntemann, B. Lipphardt, C. Tamm, V. Gerginov, S. Weyers, and E. Peik, Phys. Rev. Lett. 113, 210802 (2014).

[25] A. Windberger, J. R. C. Lpez-Urrutia, H. Bekker, N. Oreshkinan, J. Berengut, V. Bock, A. Borschevsky, V. A. Dzuba, E. Eliav, Z. Harman, et al., Phys. Rev. Lett. 114, 150801 (2015).

[26] W. R. Johnson, Canadian J. Phys. 89, 429 (2011).

[27] S. G. Porsev and A. Derevianko, Phys. Rev. A 69, 042506 (2004).

[28] V. A. Dzuba and V. V. Flambaum, Phys. Rev. A 77, 012514 (2008).

[29] P. Taylor, M. Roberts, G. M. Macfarlane, G. P. Barwood, W. R. C. Rowley, and P. Gill, Phys. Rev. A 60, 2829 (1999).

[30] A.-M. Mårtensson-Pendrill, D. S. Gough, and P. Hannaford, Phys. Rev. A 49, 3351 (1994).

[31] J. Sugar and V. Kaufman, Phys. Scr. 24, 742 (1981).

[32] M. S. Safronova, V. A. Dzuba, V. V. Flambaum, U. I. Safronova, S. G. Porsev, and M. G. Kozlov, Phys. Rev. A 90, 052509 (2014).

[33] A. Kramida, Yu. Ralchenko, J. Reader, and and NIST ASD Team, NIST Atomic Spectra Database (ver. 5.3), [Online]. Available: http://physics.nist.gov/asd [2016, January 11]. National Institute of Standards and Technology, Gaithersburg, MD. (2015).

[34] M. S. Safronova, V. A. Dzuba, V. V. Flambaum, U. I. Safronova, S. G. Porsev, and M. G. Kozlov, Phys. Rev. A 90, 042513 (2014).

[35] J. Leute, N. Huntemann, B. Lipphardt, C. Tamm, P. B. R. Nisbet-Jones, S. A. King, R. M. Godun, J. M. Jones, H. S. Margolis, P. B. Whibberley, et al., arXiv preprint arXiv:1507.04754 (2015). 